\title{
Current Status and Trends of the Research on
}

\section{Chinese-Foreign Cooperation in Running Schools}

\section{- A Statistical Analysis Based on CSSCI Papers Between 2008 and 2017}

\author{
Jing Wang* \\ International Business School \\ Yunnan University of Finance and \\ Economics \\ Kunming, China \\ 120159185@qq.com
}

\author{
Xinzhi Xi \\ School of Foreign Languages and \\ Culture \\ Yunnan University of Finance and \\ Economics \\ Kunming, China \\ 190942317@qq.com
}

\author{
Qian Zeng \\ International Office \\ Yunnan University of Finance and \\ Economics \\ Kunming, China \\ bmwii@foxmail.com
}

\begin{abstract}
This study is carried out based on 186 papers published between 2008 and 2017. The sample papers are all titled with "Chinese-foreign Cooperation in Running Schools (CCRS)", and published in journal listed in Chinese Social Science Citation Index (CSSCI). A quantitative analysis of the distribution of the years of publication, publishing journals, authors, affiliation institutes, and research topics is made to unveil the current research status. Through the statistical research, it finds that CCRS has gained increasing attention from China`s academic circle each year, and the research focuses are shifted subjecting to the change of national strategies. But the research also noticed that the main methods adopted by the previous studies are mostly qualitative based and there are only few stable research teams or platforms indicating the lack of cooperation among the researchers. Thus, this paper suggests that future research on CCRS should employ more diversified research methods, strengthen cross-disciplinary cooperation among researchers, and set itself in the historical background, so as to make a breakthrough in both research quantity and quality, and provide a complete and theoretical guidance for the practice of CCRS.
\end{abstract}

Keywords: China-foreign Cooperative Run School, CSSCI Index, higher education internationalization

\section{INTRODUCTION}

Chinese-foreign Cooperation in Running Schools (CCRS) is a fast growing model of Cross-board education in China since 1990 s and is attracting increasing attention of the scholars in China.

There are two major types of CCRS depending on the different mode of cooperation. The first one is very common and known as the Chinese-foreign cooperative programs (hereinafter called CCRS Programs), and the second one is more sophisticated and known as the Chinese-foreign cooperative institute (hereinafter called CCRS Institute). According to the official statistics issued by Ministry of Education of China, for CCRS in Higher education sector, there are 88 CCRS Institutes and 1024 CCRS Programs are approved and established in China by August 2018[1]

Due to the large number of CCRS, it has attracted much attention of the scholars in resent year. Publicly published academic papers are the best clue for unveiling the latest development of a certain research field. In order to clearly depict the latest research status and predict the future research trend of CCRS in China, an accurate and scientific analysis of the high-level papers of CCRS is conducted. The research is based on the academic papers indexed in Social Sciences Citation Index (CSSCI) including both source journals and the expanded edition, which are highly recognized and influential in China. To target on the relevant academic papers, only the papers with titles including the phrase of "Chinese-foreign cooperation in running schools" and published between 2008 and 2017 are selected. 198 samples were retrieved in total, including 12 non-academic papers. Therefore, the valid samples are 186 academic papers. By taking the quantitative and qualitative approaches, this article analyzes the sample papers from various perspectives including but not limited to the annual distribution, publishing journals and distribution of the authors. The research result will present an objective view of the current situation of the theoretical research on CCRS, and provide a clue to predict the future trend.

\section{ANALYSIS OF CURRENT RESEARCH}

\section{A. Annual distribution}

By allocating the 186 sample papers to their publishing years, it is clear that the annual output of articles related to CCRS in the recent 10 years is increasing steadily. It suggests that studies in this field are catching more and more attention of the scholars. In July 2010, The Outline of Middle and Long Term Educational Reform and Development Plan (2010-2020) was promulgated by the central government, and the document clearly proposed to "introduce high-quality foreign educational resources, and explore diverse ways to make full use of them"[2]. The document has clearly set new requirements and goals for CCRS and motivated higher education institutions to set internationalization as their development strategy one after another. As a result, the number of published CCRS related papers were obviously rising. After year 2011(except for 2016), the output of CSSCI indexed papers were over 20 per year and 
all touched wider areas and involved more diversified perspectives (See Table 1).

TABLE I. ANNUAL DISTRIBUTION

\begin{tabular}{|l|l|l|l|l|l|l|l|l|l|l|}
\hline Year & 2008 & 2009 & 2010 & 2011 & 2012 & 2013 & 2014 & 2015 & 2016 & 2017 \\
\hline Papers & 14 & 17 & 18 & 13 & 26 & 20 & 23 & 21 & 13 & 21 \\
\hline
\end{tabular}

\section{B. Distribution of journals}

An analysis of source journals often show how much attention the CCRS arouses among the academic circles. The study reveals that all the 186 sample papers are published in 65 journals. There are 10 journals which have cumulatively published more than 5 papers (See Figure 1). The total number of papers published in these 10 journals amounts to 103, accounting for $55.4 \%$ of the total. The Top five high-output journals are Heilongjiang Researches on Higher Education, China Higher Education Research, Educational Research, Journal of National Academy of Education Administration, and Jiangsu Higher Education, which have published 68 papers in total, with an average of over 10 for each, taking up $36.6 \%$ of the total. It is clear that on the one hand studies related to CCRS have several stable publishing platforms to demonstrate the latest research results and have received high attention from some high-level journals. While on the other hand, the total number of high-quality papers and journals is still low compared with over 2000 China-foreign cooperative-run institutes and programs.

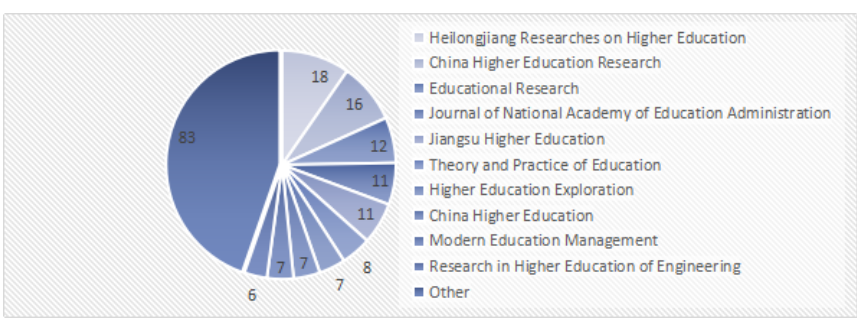

Fig. 1. Distribution of journals

\section{Distribution of authors}

Administrative staffs in higher education institutions, university teachers, and researchers are the main force for the research of CCRS. Therefore, universities and educational research departments are the main sources where the papers came from. In terms of authors, among the 186 sample papers, 100 of them are completed by only one author, which takes up $53.67 \%$. 71 of them were co-written by two authors, accounting for $38.17 \%$. Only 4 papers were finished by 3 or more authors and it accounts for $8.06 \%$. The above data indicates a low frequency of cooperation among the researchers, or a comparatively isolated research pattern adopted among the authors. Even in the coauthored papers, most of the authors are from the same institution. Cooperation and communication across institutions and regions are rarely seen. It is obvious that this kind of comparatively isolated or inbreeding-alike research is not good for academic excellence.
From the individual author perspective, only 7 scholars have published more than 3 papers. They are Jinhui Lin (11), Qiang Guo (6), Mengjin Liu (4), Weiyang Xue (4), Shengbing Li (4), Yanzhi Zhao(3) and Weiping Wang (3). Their publications accounted for $18.82 \%$ of the total (see Figure 2). Among them, Jinhui Lin(Director of Center of Research on Chinese-Foreign Cooperation in Running Schools, Xiamen University), Mengjin Liu and Shengbing Li have established their research institute or a research team engaged in the research of CCRS. The research institutes and teams led by the above scholars function as stable and sustainable platforms for the research. Although more and more CCRS practitioners and scholars have been positively involved in the academic research on CCRS in recent years which has diversified the research through different perspectives, the lack of cooperation and sharing may confine their thoughts and creativity. A tighter and closer link among the researches and more stable research teams are needed to keep the research more sustainable and more objective.

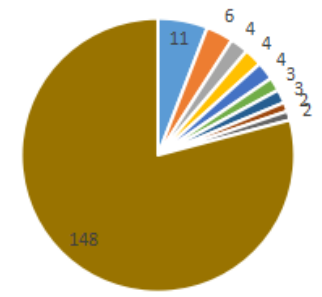

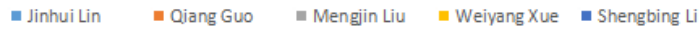

- Yanzhi Zhao - Weiping Wang - Lihui Xie - Tao Meng - Other

Fig. 2. Distribution of authors

Relying on its Center of Research on Chinese-Foreign Cooperation in Running School, Xiamen University obviously shows a great advantage over other top ten institutions in terms of the number of paper published. According to the data, authors from Xiamen University have altogether published 27 papers, accounting for $14.5 \%$ of the total, and the above number almost equals the accumulated number of papers published by the following top 4 institutes. The analysis also shows that higher education institutions are absolutely the main force in research on CCRS. The increasing amount of research on this topic reveals that as the higher education in China gets more and more internationalized, CCRS shall become a more important method to achieve the goal of internationalization. How to deepen the cooperation and make new achievements has become an unavoidable question for both the administrators and teachers of CCRS. This requires not only courage for exploration and persistence for practice, but also extensive and profound theoretical research [3].

\section{Distribution of research topics}

The analysis of the collection of keywords of a certain academic field over a long period of time can reveal the characteristics of the research achievements, the linkage between research contents, the evolution of the research and the research trends Wenlan Li, Zuguo Yang, "A frequency analysis of key words in papers on Chinese information science 
journals," in Information Science, vol. 01, 2005, pp. 68-70+ 143.]].The frequently shown keywords will highlight the research focus of a certain academic field [4]. Among the keywords of the 186 sample papers, 12 keywords have been shown for no less than 10 times (see Figure 3). The frequencies of those keywords are listed in descending order as follows: CCRS programs (105), internationalization of education (45), CCRS institution (40), regulations on CCRS (38), CCRS operators (24), foreign teacher (19), talent cultivation (19), cooperative modes (15), educational cooperation (13), teaching effect (12), international talents (12), and study abroad (12). All the 12 keywords can be divided into two categories, namely "CCRS Programs and Institutes" from the macro perspective, and "talent cultivation" from the micro perspective. The first category is the mainstream of the research, which focuses on how to run the China-foreign cooperative programs or institutes. The specific topics include the cooperation mode, relevant policies and regulations, internationalization and qualification of the operators. The second category can be described as student-centered research. The specific topics include teaching force, teaching quality assessment, internationalized education, and student beneficiaries. On the whole, as CCRS develops in China, the scope of the relevant research also expends. Although the enlarged research scope shall have a positive effect on promoting CCRS, some issues need to be noted. The majority of the current studies mainly focus on the analysis of current situation, but studies on some essential problems, such as quality enhancement, management regulation and connotation improvement are not sufficiently highlighted yet.

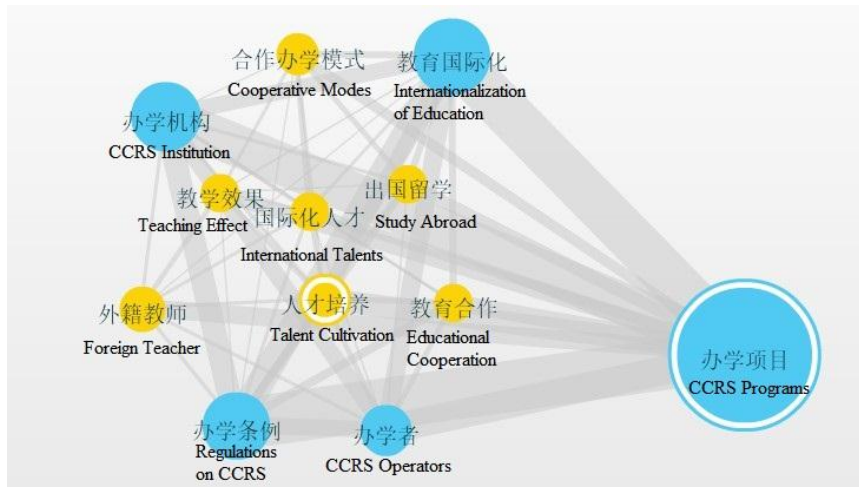

Fig. 3. Linkage Network of the Keywords

\section{RESEARCH TRENDS}

In summary, studies on CCRS have made substantial progress not only in quantity and quality, but also in scopes and depth. The research findings are also valuable and helpful for guiding the practice of the CCRS. However, it is easy to find that the research does not form up its own research system. In the future, more systematic and forward-looking studies and thinking on CCRS are needed because it will not only enrich theoretical findings, but also be of great importance to steer the directions of CCRS practices.

\section{A. Strengthen basic research, and integrate into national strategy}

On the one hand, though researches on CCRS keep increasing each year, most of them are general studies only. That is to say, most of them either focus on explaining what CCRS is and how to carry it out, or emphasize the significance and effects. Few of them pay attention to the laws behind it, or in other words, the essential problems such as why and how it is so. As a result, in-depth studies on the nature and sustainable development of CCRS are few. While on the other hand, one of the general purposes of the scientific research is to connect theory with reality and eventually promote the economic and social development. At present, China is carrying out the "Belt and Road" Initiative. Hence, future research on CCRS should be conducted under and for this "umbrella". To be specific, studies of the educational environment, policies and market of the "Belt and Road" countries should be enriched and enhanced. This will not only provide constructive suggestions for the development of CCRS under the background of "Belt \& Road" Initiative, but also provide intellectual support for the innovative development of CCRS in the "New Era" as mentioned by General Secretary Xi Jinping.

\section{B. Follow the "Introduce-Absorb-Output" pattern}

As China is such a huge country with vast territory, its huge differences in economy, society and culture across the country and across borders will definitely affect the development of CCRS. Therefore, current studies have shifted from the stage of paving the theoretical ground of CCRS by learning from various international education concepts and practices to a new stage which is featured by absorbing the good practices and localizing the "imported" thoughts and concepts. Localization and customization of CCRS in return will provide diversified samples for researchers. Researchers should put more attention on the uniqueness of each CCRS program or institute, and find ways to prevent homogenization and cut-throat competition.

Furthermore, both CCRS and "Overseas Campus" belong to the concept of "Cross-Border Education [5]. The only difference is that the former one is inbound oriented and the later one is outbound oriented. Most of the Chinese universities have already accumulated much experience in either CCRS, or how to import advanced foreign educational resources. On the contrary, only a few of them have set up degree programs or established overseas campuses in other countries. Since the CCRS and overseas campuses are two sides of one coin, they share great similarities. As more and more Chinese universities intend to involve in exporting their education, researches on CCRS will be helpful for them and eventually stimulate the research on overseas campuses or exporting of education.

\section{Strengthen synergistic innovation, and integrate research forces}

Individual researchers often turn out to be weak and lonely, so do the researchers of the CCRS. There is little cooperation among the authors, especially between theoretical researchers and practitioners, education orientated researchers and noneducation orientated researchers. The number of research platforms, teams, institutes and associations for sharing thoughts, seeking cooperation and brainstorming ideas are still far from enough. Even if there are some cooperation, the co- 
authors are not stable, which means most of the research cooperation is occasional and short-termed. The above lack of cooperation or low level of cooperation is the existing obstacle for improving the quantity and quality of the CCRS research and forming strong research teams in the long turn. Creating a favorable ecological environment for the scientific research and motivating synergistic innovation and trans-disciplinary cooperation are the key issues that the government and researchers should cope with in the future.

\section{Stress quantitative research and improve research paradigms}

Generally speaking, although CCRS is a very practical and comprehensive activity in higher education internationalization, researchers tend to consider it as a social phenomenon and seldom take diversified research approaches. Among the sample papers, nearly $87 \%$ of the studies choose to take a qualitative approach and this was noticed long before by Cheng Bin [6]. The research also found that even if there were some quantitative or other kind of research approaches, they were merely imitating and the researchers often paid little attention to research methods and theoretical basis. These weaknesses in current researches will become barriers for CCRS research to be more systematic, theoretical and diversified [7]. Future studies should adopt more research approaches including but not limited to field studies, case study, quantitative study and empirical study. It is also suggested to take perspectives from different disciplines such as education, management, law, sociology, and economics and so on. The improvement of research approaches will not only promote and synergize the theoretical research and practical activities of CCRS, but also cultivate a group of capable researchers and teams.

\section{ACKNOWLEDGMENT}

First of all, I would like to extend my gratitude to my coauthors for their dedicated work and support from the very beginning to the completion of this paper.

I also would like to thank Yunnan Provincial Department of Education, which supported this research by granting a research project (No.SYSX201920) to one of the coauthor to carry out the relevant research.

Lastly, I offer my regards to all of those who supported us in any respect during the completion of this work.

\section{REFERENCES}

[1] http://www.jsj.edu.cn/news/2/1184.shtml

[2] http://old.moe.gov.cn/publicfiles/business/htmlfiles/moe/info_list/20140 7/xxgk_171904.html

[3] Jianhua $\mathrm{Hu}$, "The importance of theory research to the practical development of Chinese-foreign cooperation in running schools --Comments on Chinese-foreign cooperation in running schools in higher education," in Educational Research, vol. 10, 2010, pp. 105.

[4] Xiufen An, Xiaoli Huang, Xia Zhang, Chaoying Lin, "An analysis of key words in academic papers on literature metrology in journal work," in Chinese Journal of Scientific and Technological Periodicals, vol. 13(06), 2002, pp. 505-506.

[5] OECD. Cross-border education: An overview, OECD/Norway forum on trade in educational services: Managing the internationalization of postsecondary education[R].2003.

[6] Bin Cheng, Yikang Cheng, "A general survey on Chinese-foreign cooperation in running schools since 2000," in Academic Forum, vol. 2, 2007, pp. 199.

[7] Jianmin Tang, "Research history of non-governmental higher education in the recent decade - a bibliometric analysis of research papers between 1999 and 2008 and recognition based on visualization," in Modern University Education, vol. 2, 2009, pp. 33. 